\title{
UTILIZACIÓN DE QUELONIOS DE INTERÉS ECONÓMICO EN EL MUNICIPIO DE CAIMITO, SUCRE, COLOMBIA
}

\section{USE OF CHELONIANS OF ECONOMIC INTEREST IN THE MUNICIPALITY OF CAIMITO, SUCRE, COLOMBIA}
DE LA OSSA, V. JAIME ${ }^{1 *}$ Dr., OLIVERO-GÓMEZ, GRACE² Zootec., RUIZ. JOSE GREGORIO² Zootec.,

$1^{*}$ Profesor Titular Universidad de Sucre, Facultad de Ciencias Agropecuarias, Colombia. Grupo de Investigación en Biodiversidad Tropical. Sincelejo, Sucre, Colombia. $^{2}$, Zooctenistas Universidad de Sucre, Colombia.

*Correspondencia: jaimedelaossa@yahoo.com

Recibido: 16-04-2010; Aceptado: 06-11-2010.

\section{Resumen}

En el trabajo se evaluaron los patrones de utilización de quelonios de interés económico en el municipio de Caimito, departamento de Sucre, Colombia, durante los meses de septiembre a octubre del 2008. Se utilizaron diálogos interactivos con miembros de la comunidad en general para identificar a los pobladores que se dedican a la cacería, comercio y/o consumo de quelonios de interés económico, a quienes se les aplicaron encuestas para recopilar la información biológica sobre la disponibilidad y el uso de los quelonios cazados. Se trabajó con una muestra representativa de 90 cazadores y/o comerciantes de quelonios o fauna silvestre identificados previamente con los diálogos interactivos. El quelonio de mayor uso en el municipio de caimito es Trachemys callirostris (hicotea), se venden vivas con tamaños promedios de $13,93 \mathrm{~cm} / \mathrm{ind}$ (longitud total del caparax), pesos aproximados de 1072,22 gr/ind y valores promedios de $\$ 4.194,44$ por unidad. Las personas dedicadas a estas actividades utilizan alrededor de 13.644 ind/año, de los que para la venta disponen más del $40 \%$ del total de los individuos capturados, los cuales le generan ingresos entre 0,151 y 0,242 S.M.M.L.V. (Salario Mínimo Mensual Legal Vigente).

Palabras clave: quelonios, utilización, comercio, Caimito, Sucre, Colombia.

\begin{abstract}
This study evaluated the chelonian's utilization patterns with economic interest in Caimito municipality, Sucre department, Colombia, during September to October 2008, using interactive dialogues with community members in general to indentify the people engaged in hunting, trade and consumption of chelonians with economic interest, who were given surveys to collect biological information on the availability and use of Chelonians hunted it was worked with a representative sample of 90 hunters of chelonians or wildlife, previously
\end{abstract}


identified with the interactive dialogues. The greatest use of chelonians in Caimito municipality is Trachemis callirostris (slide turtle), they are sold live with average size of $13.93 \mathrm{~cm}$ (total caparax length), approximate weight of 1072.22 $\mathrm{g}$ and average values of $\$ 4194.94$ per unit. People engaged in these activities use about $13,644 \mathrm{ind} /$ year, which have sold for more than $40 \%$ of all individuals captured, which generate earnings between 0.151 and 0.242 current legal monthly minimum wage.

Key words: chelonians, utilization, incomes, Caimito, Sucre, Colombia.

\section{Introducción}

La fauna silvestre es uno de los recursos naturales renovables básicos, junto al agua, el aire, el suelo y la vegetación. Las especies nativas en su conjunto constituyen la riqueza y diversidad genética de los ecosistemas y forman parte del patrimonio natural de países, regiones y del mundo (OJASTI, 2000). Desde épocas precolombinas hasta la actualidad la fauna silvestre ha ocupado una posición fundamental en el desarrollo de los grupos sociales colombianos tanto en términos simbólicos como materiales (BAPTISTE et al., 2009).

En Colombia la explotación de la fauna silvestre con fines económicos ha venido generando un gran impacto sobre las poblaciones naturales, específicamente sobre aquellas que tienen una fuerte demanda cultural, ya sea para usos medicinales o gastronómicos, hasta el punto que algunas de ellas han sido llevadas al borde de la extinción (BAPTISTE et al., 2009).

Nuestro país posee aproximadamente el 10\% de fauna y flora existente en el planeta, lo cual lo sitúa como uno de los más ricos en diversidad biológica; al igual que ocupa el cuarto puesto en número de reptiles (SÁNCHEZ et al., 1995). Por su parte, los reptiles han sufrido una drástica reducción en sus poblaciones naturales, producidas por la caza indiscriminada, la destrucción de sus nidos y la fragmentación de los hábitats, que son en esencia acciones antrópicas (DE LA OSSA y RIAÑO, 1999).

Las tortugas son y siguen siendo usadas como alimento, mascotas y medicina en todo el mundo (BATES, 1963; BRITO y FERREIRA, 1978; ALHO, 1985; AYRES et al., 1991; GOULDING et al., 1996; CANTARELLI, 1997; PERES, 2001; FACHIN-TERÁN et al., 2004). Durante las últimas décadas, las tortugas han sufrido una drástica disminución de sus poblaciones, debido a la destrucción de su hábitat, caza, comercio de huevos para consumo humano y venta de neonatos o juveniles para mascotas, así como por el aumento de la depredación de huevos, juveniles y adultos. En general las tortugas son un componente de gran valor en la dieta de los indígenas y habitantes en general 
(FERREIRA, 1786; LA CONDOMINE, 1992). No obstante, no se tiene en la actualidad información cuantitativa sobre los niveles de cosecha de quelonios y los efectos de esta actividad sobre las poblaciones naturales.

Un punto importante en la conservación de los quelonios debe ser el conocimiento de los patrones de uso, el efecto de ellos sobre las poblaciones naturales, su importancia para la subsistencia comunitaria y como la utilización y el valor intrínseco de las tortugas puede usarse como herramienta para construir proyectos sustentables (MITTERMEIER, 1992; PINEDO-VASQUEZ et al., 2002); apoyando necesariamente este conocimiento sociocultural con investigaciones biológicas básicas y ecológicas fundamentales. Trachemys callirostris según IUCN (2010) se cataloga como de bajo riesgo pero cercana a amenazada (NT) desde 1996.

En este estudio se presenta información cuantitativa y cualitativa relacionada con la utilización de quelonios de interés económico en el área jurisdiccional del municipio de Caimito, Sucre, Colombia; se resalta el valor social del recurso como factor cultural y de subsistencia, al tiempo que se evidencia como la especie de mayor presión a Trachemys callirostris (hicotea).

\section{Materiales y Métodos}

Área de estudio: El presente trabajo se realizó durante los meses de septiembre a octubre de 2008, en el municipio de Caimito, departamento de Sucre, ubicado en la subregión San Jorge a $8^{\circ} 47^{\prime} 35^{\prime \prime} \mathrm{N}$ y $75^{\circ} 23^{\prime} 34^{\prime \prime}$ O (Fig. 1), región que forma parte de los grandes humedales de la Mojana. Este municipio tiene una extensión de $428 \mathrm{~km}^{2}$, con una población aproximada de 10.195 habitantes, temperatura media de $28 \stackrel{\circ}{\circ}$, a una altura de $25 \mathrm{msnm}$. El Municipio pertenece, según HOLDRIDGE (1967) a la formación bosque seco tropical (bs-T); puede igualmente ser identificado como un zonobioma tropical alternohigríco (HERNÁNDEZ y SÁNCHEZ, 1992); caracterizado por clima cálido seco (IGAC, 1969).

Colecta de información: Se aplicaron encuestas estructuradas siguiendo las consideraciones descritas por MARGOLUIS y SALAFSKY (1998); para recopilar la información biológica sobre la disponibilidad y el uso de los quelonios cazados; se trabajó con una muestra representativa equivalente al $50 \%(\mathrm{~N}=90)$ de los cazadores $\mathrm{y} / \mathrm{o}$ comerciantes de quelonios o de fauna silvestre en general, que fueron identificados en los diálogos previamente descritos.

El cálculo de la captura se hizo mediante registro diario de los animales obtenidos por cada cazador; los datos relacionados con la morfometría de las 
capturas se basó en la elección al azar de una muestra de 100 individuos por rango de talla; y La medida de esfuerzo utilizada fue determinado por el número de individuos capturados por especie con relación al tiempo empleado: (Esfuerzo de Caza) $\mathrm{EC}=\mathrm{NIC} / \mathrm{T}$ : donde, $\mathrm{NIC}=$ Número de individuos capturados por especie, $\mathrm{T}=$ Tiempo empleados en días.

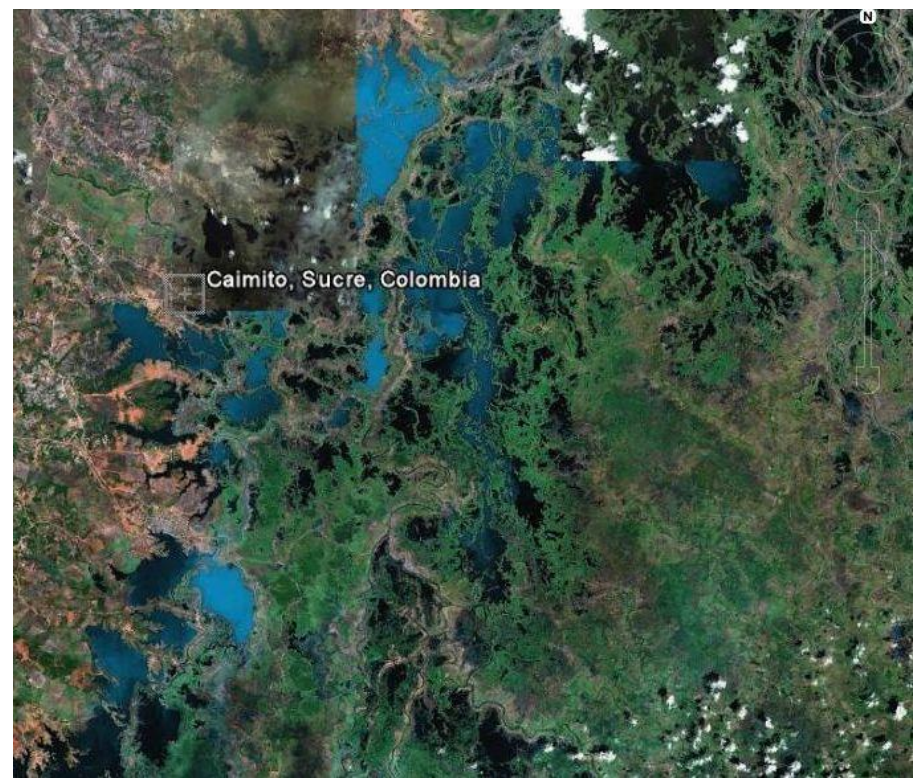

Figura 1. Mapa de ubicación del sitio de trabajo (Google Earth, versión 5.0 Free)

Análisis de información: Los datos obtenidos se organizaron y caracterizaron sistemáticamente utilizándose la estadística descriptiva. El análisis de datos cuantitativos de encuestas y registros de uso de recursos, fue analizado mediante la aplicación y representación de tablas. Para comparar las muestras se utilizaron cálculos de porcentajes y promedios. El análisis de datos cualitativos se realizó mediante un registro sistemático agrupando las respuestas según la naturaleza y el grado de concordancia con la pregunta.

\section{Resultados}

De la población participante en este trabajo se obtuvo la siguiente información: $95,5 \%$ del sexo masculino y 4,44 del sexo femenino desempeñan la caza, lo que representa $84,44 \%$ (76) para este sector, siendo los comercializadores $15,55 \%$ (14). Para la comunidad estudiada se presenta un $84,44 \%$ de analfabetismo, el carácter religioso estuvo presente en todos los encuestados. El rango de edad estuvo entre 15 y 65 años.

La preferencia de las diferentes especies de quelonios existentes en el área de estudio se presentan en la Tabla 1. Las capturas llevadas a cabo se presentan en la Tabla 2. En cuanto a captura por lugar geográfico, se halló, que las veredas La Mejía y Las Ossas son explotadas en un 32,22\% y 18,88\%, 
respectivamente; el restante $48,9 \%$ se efectúa en lugares no precisos dentro del territorio municipal inundado.

Tabla 1. Especies quelonios de interés económico por orden de preferencia en Caimito

\begin{tabular}{|l|l|c|c|}
\hline \multicolumn{1}{|c|}{ Especie } & \multicolumn{1}{c|}{ N. Común } & N & \multicolumn{1}{c|}{$\%$} \\
\hline Trachemys callirostris & hicotea & 90 & 100 \\
\hline Kinosternon scorpiodes & tacán & 49 & 54,44 \\
\hline Rhinoclemmys melanosterna & palmera, galápago & 41 & 45,55 \\
\hline
\end{tabular}

Tabla 2. Captura por especie para el municipio de Caimito

\begin{tabular}{|l|l|l|l|}
\hline \multicolumn{1}{|c|}{ Especie } & \multicolumn{1}{c|}{ N. Común } & \multicolumn{1}{c|}{$\mathbf{N}$} & \multicolumn{1}{c|}{$\%$} \\
\hline Trachemys callirostris & hicotea & 13.644 & 86,6 \\
\hline Kinosternon scorpiodes & tacán & 135 & 0,80 \\
\hline Rhinoclemmys melanosterna & palmera, galápago & 1.980 & 12,6 \\
\hline Total quelonios de interés económico & 15.759 & 100 \\
\hline
\end{tabular}

La captura discriminada por talla para $T$. callirostris se muestra en la Tabla 3.

Tabla 3. Captura discriminada por talla (LTC) para T. callirostris

\begin{tabular}{|c|c|c|c|c|c|}
\hline Talla & Media & $\mathbf{N}$ & $\mathbf{P}(\mathbf{g})$ medio & Venta (\$ Col) media & $\%$ \\
\hline$<9$ & 8,22 & 2.364 & 340 & 1.500 & 15 \\
\hline $10-15$ & 11,28 & 2.994 & 590 & 2.500 & 19 \\
\hline $16-20$ & 13,93 & 8.195 & 1.202 & 5.000 & 52 \\
\hline $21-25$ & 23,1 & 1.418 & 1.482 & 6.000 & 9 \\
\hline $25>$ & 26,7 & 788 & 1.747 & 7.500 & 5 \\
\hline & & 15.759 & 1.072 & 4.500 & 100 \\
\hline
\end{tabular}

En cuanto al consumo y venta de las quelonios cazados, se presentan los datos de Trachemys callirostris en la Tabla 4. En relación con Kinosternon scorpiodes (tacan), todos manifiestan que su captura es ocasional y que se usa como alimento para ellos mismos; mientras que Rhinoclemmys melanosterna (palmera, galápago) se consume y comercializa de igual forma que $T$. callirostris.

Tabla 4. Relación consumo Vs. venta de individuos capturados para Trachemys callirostris

\begin{tabular}{|l|l|l|l|}
\hline Consumo & Venta & $\mathbf{N}$ & $\begin{array}{c}\text { Total } \\
(\%)\end{array}$ \\
\hline 0 & 100 & 17 & 18,9 \\
\hline 20 & 80 & 17 & 18,9 \\
\hline 30 & 70 & 18 & 20,0 \\
\hline 40 & 60 & 36 & 40,0 \\
\hline 60 & 40 & 2 & 2,2 \\
\hline Total & 90 & 100 \\
\hline
\end{tabular}


La participación familiar en la utilización y/o comercialización de tortugas varía de una a tres personas (Fig. 2). El ingreso mensual está entre $\$ 75.455,55$ y \$120.555,55 que representan entre 0,151 y 0,242 Salarios Mínimo Mensual Legal Vigente (\$496900 S.M.M.L.V. para 2009) para la temporada de caza (Tabla 5).

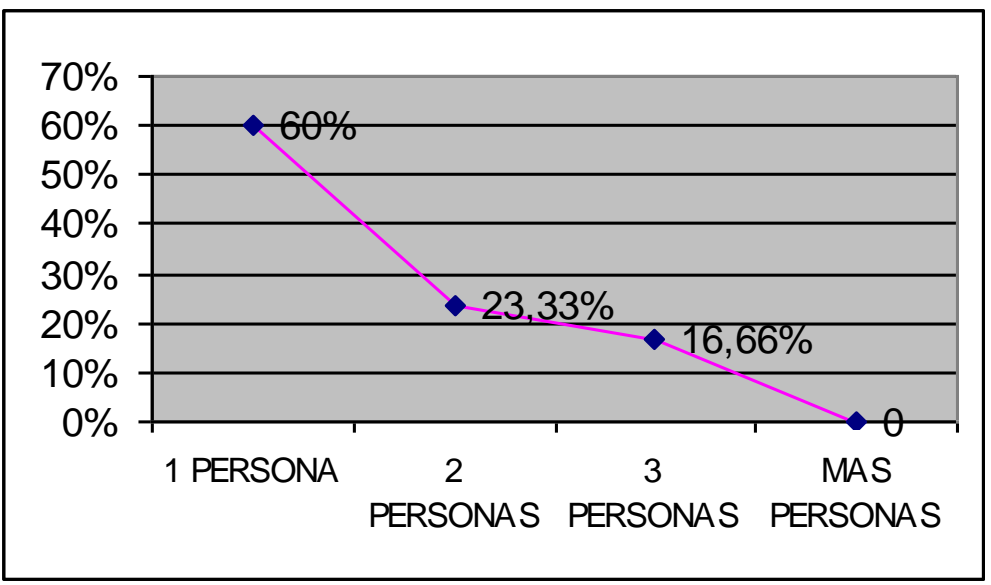

Figura 2. Participación familiar en utilización y/o comercialización de quelonios.

Tabla 5. Ingreso mensual por la venta de quelonios.

\begin{tabular}{|l|l|l|}
\hline Ingresos (\$ Col.) & $\mathbf{N}$ & \multicolumn{1}{c|}{$\%$} \\
\hline $10.000-50.000$ & 39 & 43,33 \\
\hline $51.000-100.000$ & 16 & 17,77 \\
\hline $101.000-150.000$ & 13 & 14,44 \\
\hline $151.000-200.000$ & 7 & 7,77 \\
\hline $201.000-250.000$ & 11 & 12,22 \\
\hline $251.000-300.000$ & 4 & 4,44 \\
\hline
\end{tabular}

El cálculo de esfuerzo de caza se presenta en la Tabla 6. Se realizó tomando como base una actividad anual de 5 meses (150 días), que hacen relación al primer período del año, el cual se caracteriza por inicio de la época seca, reproducción de las especies aquí relacionadas y consumo cultural ligado a cuaresma y semana santa.

Tabla 6. Esfuerzo de captura (EC) por día con referencia al período de captura anual para quelonios de interés económico en Caimito.

\begin{tabular}{|l|l|l|l|l|}
\hline \multicolumn{1}{|c|}{ Especie } & N. Común & \multicolumn{1}{c|}{$\begin{array}{c}\text { Captura } \\
\text { (ind/temporada) }\end{array}$} & $\begin{array}{c}\text { EC } \\
\text { (ind/día) }\end{array}$ & $\begin{array}{c}\text { EC } \\
\text { (ind/cazador/día) }\end{array}$ \\
\hline T. callirostris & hicotea & 13.644 & 90,96 & 1,196 \\
\hline K. scorpiodes & tacán & 135 & 0,9 & 0,012 \\
\hline R.melanosterna & palmera & 1.980 & 13,2 & 0,173 \\
\hline Total quelonios capturados & 15.759 & 105,06 & 1,382 \\
\hline
\end{tabular}




\section{Discusión}

En índice de analfabetismo detectado para la muestra de personas con que se trabajó en el presente estudio muestra un valor significativamente elevado ya que el promedio nacional determinado es de $8,6 \%$ para el año 2005, según los resultados estadísticos del censo 2005 publicados por el DANE.

La caza y/o comercialización de tortugas es una actividad de supervivencia que se complementa con otras labores productivas, como la pesca de subsistencia y la agricultura, estos coincide con lo reportado por DE LA OSSA y VOGT (2009), puede interpretarse a esta actividad extractiva como una fuente importante de recursos alimenticios y comerciales cuyo origen además de ser cultural es una consecuencia que incluye factores diversos de orden social y económico, coincidiendo con lo señalado por OJEDA y MARES (1982), MARES y OJEDA (1984), OJEDA et al. (1998), REDFORD y SANDERSON (2000), FUENTES-OBEID et al. (2003).

En el estudio se registró que $60 \%$ de las familias solo una persona participa en las actividades de caza y/o comercialización de fauna silvestre, pero los ingresos obtenida por esta actividad no superan un salario mínimo legal vigente mensual. Durante la temporada de captura; según BAPTISTE et al. (2009), la actividad puede proveer ingresos estables equivalentes a un salario mínimo mensual vigente a una familia dedicada a esta actividad, lo cual no se registra en este caso, se puede asumir que los ingresos dependen de la abundancia natural y de una buena conexión con la rede comercial. HAMES (1991); ALVARD, (1995) DEMMER et al. (2002) concuerdan en que dentro de un sistema de economía de subsistencia se manifiestan de forma contundente las consecuencias del incremento poblacional, la demanda de recursos naturales y la comercialización, creándose un impacto devastador entre las comunidades nativas y el medio ambiente, pues se puede ver al recurso fauna silvestre como una vía rápida de obtener dinero relativamente fácil.

T. callirostris en su mayoría se captura mayoritariamente con talla media de $13,93 \mathrm{~cm}$ (longitud total del caparax), pero al analizar la captura discriminada se observa que $34 \%$ se ubican con certeza como individuos inmaduros, en tal sentido MEDEM (1975), señala que alcanzan la madurez a los tres años de edad con longitud total del caparax de $15,8 \mathrm{~cm}$ para hembras y $12,00 \mathrm{~cm}$ para los machos; se puede deducir que la captura con talla media de 13,93 incluye animales de primera postura y que su representativa captura está relacionada con la estación reproductiva; igualmente es importante anotar que como individuos maduros acorde con la talla se tendría solo $13 \%$, que representativamente es un valor bajo y pondría en evidencia una deterioro 
poblacional por extracción masiva, información que se ajusta a lo establecido por FUENTES-OBEID et al. (2003).

El peso promedio de la captura total es de $1.072 \mathrm{gr}$ con valor individual aproximado de $\$ 4.500$, que según los comerciantes de zona es bajo al compararlos con los precios de la época de mayor demanda donde el valor por individuo llegar hasta $\$ 15.000$ en el mercado local acopiador. Las ganancias de los comerciantes siempre son mayores y significativamente difieren de las obtenidas por lo cazadores como en este caso; estando de acuerdo con lo señalado por PEREZ (2000), quien resalta la importancia de las comunidades naturales y las relaciona con su productividad y los efectos negativos que la caza de subsistencia pueden llegar a generar; reconociéndose al mismo tiempo lo planteado por BOLTON (1997); NOSS (2000), quien indica que la caza de subsistencia, va más allá de la comunidad y sus efectos se observan gracias al mercado, que no práctica la selectividad y se aprovecha de la extracción, ya que cualquier animal que sea cazado si no es vendido es consumido.

En cuanto al esfuerzo de caza, se evidencia una captura individuo/día que no alcanza los 2 ejemplares en $T$. callirostris, como mayor quelonio colectado en la zona, lo que muestra un esfuerzo elevado en función de las ganancias obtenidas, pero que además permite observar complementariamente que las poblaciones están declinando localmente. REDFORD y SANDERSON (2000), indican que los efectos ejercidos por las comunidades son el resultado de fuertes y negativas presiones de orden social, económico y ambiental, en este caso el extractivismo básico como alternativa de subsistencia puede llevar a la extinción local y luego a la extinción total (MOCKENHAUPT, 1999; GIBBONS et al., 2000).

Como en este caso, se debe considerar que las comunidades rurales, especialmente aquellas aisladas dependen de abundantes recursos de caza para mantener un buen nivel nutricional, especialmente en las épocas en que existe escasez por factores de ambientales de la pesca o de la agricultura familiar, concordando con VICKERS (1984), REDFORD y ROBINSON (1987), STEARMAN (1990), TOWNSEND (1996), BENNETT y ROBINSON (2000), ROBINSON y BENNETT (2000).

\section{Referencias}

ALHO, C.J.R. 1985. Conservation and management strategies for commonly exploited amazonian turtles. Biological Conservation 32(2):291-298.

ALVARD, M. 1995. Shotguns and subsistence hunting in the Neotropics. Oryx 29:58-66. 
AYRES, J.M.; Magallhaes, E.; Souza, M.; Barreiros, J.L. 1991. On the track of the road: changes in subsistence hunting in Brazilian Amazon village. Págs.8292. En: Robinson, J.G.; Redford, K.H. (eds.). Neotropical Wildlife Use and conservation. Chicago University Press. Chicago.

BAPTISTE, L.G.; HERNÁNDEZ, S.; POLANCO, R.; QUICENO, M. P. 2009. La fauna silvestre colombiana: una historia económica y social de un proceso de marginalización. Disponible en:

http://www.humboldt.org.co/pdf/usoyval/Baptiste.pdf. Consultado: 19-04-2009.

BATES, H.W. 1963. The naturalist on the river Amazons. Joh Murray, London.

BENNETT, E.L.; ROBINSON, J.G. 2000. Hunting for sustainability: the start of a synthesis. Págs. 499-519. En: Robinson, J.G.; Bennett, E.L. (eds.). Hunting for Sustainability in Tropical Forests. Columbia University Press, New York.

BOLTON, M. 1997. Conservation and the use of wildlife resources. CAMPFIRE. 1999. Disponible en: Campfire Association Zimbabwe. Consultado 12 de enero de 2006.

BRITO, W.L.; FERREIRA, M. 1978. Fauna Amazônica preferida como alimento. Uma analise Regional. Brasil florestal 9(35):11-17.

CANTARELLI, V.H. 1997. The Amazon Turtles: Conservation and Management in Brasil. Págs. 407-410. En: Van Abbena, J. (ed.). Proc. Conservation, Restoration of tortoises and turtles. An International Conferences. Turtle and Tortoise Society, State University of New York, New York.

DE LA OSSA, V.J.; VOGT, R. 2009. Efecto de sustitución: una expresión del agotamiento poblacional de quelonios en Barcelos, Amazonas, Brasil. Rev. Asoc. Col. Cienc. Biol. 21(1):143-154.

DE LA OSSA, V.J.; RIAÑO S.R. 1999. Guía para el manejo, cría y conservación de la hicotea o jicotea Trachemys scripta callirostris (Gray). Convenio Andrés Bello. Ciencia y tecnología. № 74, Santa Fe de Bogotá.

DEMMER, J.; GODOY, R.; WILKIE, D.; OVERMAN, H.; TAIMUR, M.; KARIN, F.; GUPTA, G.; MCSWEENEY, K.; BROKAW, N.; SRIRAM, S.; PRICE, T. 2002. Do levels of income explain differences in game abundance? An empirical test in two Honduran villages. Biodiversity and Conservation 11:18451868. 
FACHIN-TERÁN, A.; VOGT, R. C.; THORBJARNARSON, J. 2004. Patterns of use and hunting of turtles in the Mamirauá Sustainable Development Reserve, Amazonas, Brasil. Págs. 362-77. En: Silvius, K.M; Bodmer, R.; Fragoso, R. (eds.). Nature Wildlife conservation in South and Central America. Columbia University Press, New York.

FERREIRA, A.R. 1796. Viagem filosófica pelas capitaniaza de Grão Pará, rio Negro, Mato Grosso, e Cuibá. Memórias: Zoología, Botânica. Reprint Conselho Federal de Cultura. Rio de Janeiro.

FUENTES-OBEID, S.; SAMPEDRO-MARÍN, A.; ARDILA-MARULANDA, M. 2003. Importancia de la jicotea (Trachemys scripta callirostris: Chelonia, Emydidae) como recurso natural en la comunidad de Isla del Coco, Región de La Mojana, departamento de Sucre, Colombia. Revista Biología17 (2):126-133.

GIBBSONS, J.W.; SCOTT, D.E.; RYAN, T.J.; BUHLMANN, K.A.; TUBERVILLE, T.D.; METTS, B.S.; GREENE, J.L.; MILLS, T.; LEIDEN, Y.; POPPY, S.; WINNE, C.T. 2000. The global decline of reptiles, dèjà vu amphibian. Bioscience 50:653-666.

GOULDING, M.; SMITH, N.J.H.; MAHAR, D.J. 1996. Floods of fortune: ecology and economy along the Amazon. Columbia University Press, New York.

HAMES, R. 1991. Wildlife conservation in tribal societies. Págsw. 172-199. En: Oldfield, M.L.; Alcorn, J. (eds.). Culture, Conservation and Ecodevelopment, Boulder: Westview Press..

HERNANDEZ, C.J.; SÁNCHEZ, E. 1992. Biomas terrestres de Colombia. Págs. 153-174. En: Halffter, I.G. (ed.). La Biodiversidad Biológica de Iberoamérica CYTED, Inst. De Ecología y Secretaria del Desarrollo Social. México.

HOLDRIDGE, R.L. 1967. Life zone ecology. Tropical Science Center. San José de Costa Rica.

IGAC-INSTITUTO GEOGRAFICO AGUSTIN CODAZZI. 1969. Monografía del departamento de Sucre. Oficina de Estudios Geográficos. Bogotá.

IUCN. 2010. Red list of threatened species. Disponible en: Trachemys scripta (Common Slider, Cumberland Slider Turtle, Red-eared Slider Turtle, Slider, Yellow-bellied Slider Turtle). Consultado: 05-10-2010.

LA CONDOMINE, C.M. 1992. Viagem pelo Amazonas 1735-1745. Univ. De São Paulo. Edit. Nova Fronteira. Río de Janeiro. 
MARES, M.A., OJEDA, R.A. 1984. Faunal commercialization as a factor in south American rarefaction. Bioscience 34:580-584.

MARGOLUIS, R.; SALAFSKY, N. 1998. Measures of success: designing, managing, and monitoring conservation and development projects. Island Press. Washington, DC.

MEDEM, F. 1975. La reproducción de la hicotea. Caldasia 11(53/75):83-106.

MITTERMEIER, R.A. 1992. A new species of marmoset, genus Callithrix Erxleben, 1777 (callithrichidae). Pri Geldiana Zoologia 14:1-17.

MOCKENHAUPT, B. 1999. Turtles can't hide from hungry humans, Cambodian Daily:1-2.

NOSS, A.J. 2000. Cable snares in the Central African Republic. Págs. 282-304. En: Robinson, J.G.; Bennett, E.L. (eds.). Hunting for Sustainability in Tropical Forests. Columbia University Press, New York.

OJASTI, J. 2000. Manejo de fauna silvestre neotropical. SIMAB series \# 5. Smithsonian Institute. Programa MAB. Washington DC.

OJEDA, R.A.; CAMPOS, C.M.; GONNET, J.M.; BORGHI, C.E.; ROIG, V.G. 1998. The Mab nreserve of Nacũn'an, Argentina: its role in Understanding the Monte Deser biome. Journal of Arid Environments 39:299-313.

OJEDA, R.A.; MARES, M.A. 1982. Conservation of South American mammals: Argentina as a paradigm. Mammalian Biology in South America (Serie 6). Pymatuning Lab. Ecology. Linnesville, PA.

PERES, C. A. 2001. Synergistic effect of subsistence hunting habitat fragmentation on Amazonian forest vertebrates. Conservation biology 15(6): 1490-1505.

PERES, C.A. 2000. Effects of Subsistence Hunting on Vertebrate Community Structure in Amazonian Forests. Conservation Biology 14(1):240 -253.

PINEDO-VASQUEZ, M.; BARLEHI, P.J.; DEL CASTILLO, T.D.; COFFY, K.A. 2002. tradition of change: the dinamic relatioship between biodiversity and society in sector Muyuy, Perú. Environ. Sci. And Polic. 5(1):43-53.

REDFORD, K.; ROBINSON, J. 1987. The game of choice. Patterns of Indian and colonist hunting in the neotropics. American Anthropologist 89(3):213-223. 
REDFORD, K.H; SANDERSON, S. 2000. Stracting human from nature. Conservation Biology 14(5):1362-1364.

ROBINSON, J.G.; BENNETT, E.L. 2000. Carrying capacity limits to sustainable hunting in tropical forests. Págs. 13-30. En: Robinson. J.G.; Bennett, E.L. (eds.). Hunting for sustainability in tropical forests. Columbia University Press, New York.

SÁNCHEZ, H; CASTAÑO, O; CÁRDENA, G. 1995. Diversidad de los reptiles en Colombia. Págs. 227-326. En: Rangel, O (ed.) Colombia Diversidad Biótica I. Convenio Inderena - Universidad Nacional de Colombia. Bogotá DC.

STEARMAN, A.M. 1990. The effects of settler incursion on fish and game resources of the Yuqui, a native Amazonian society of eastern Bolivia. Human Organization 49:373-385.

TOWNSEND, W.R. 1996. Nyao Itõ: Caza y pesca de los Sirionó. Universidad Mayor de San Andres, La Paz, Bolivia.

VICKERS, W.T. 1984. The faunal components of lowland South American hunting kills. Interciencia 9:366-376. 